\title{
CpG Island Methylator Phenotype(CIMP) is An Independent Prognostic Marker with Tumor-Promoting Functions in Colorectal Cancer
}

\author{
Jiante Li \\ Wenzhou Medical University Second Affiliated Hospital \\ Kepeng Hu \\ Wenzhou Medical University \\ Chenchen Wu \\ Wenzhou Medical University Second Affiliated Hospital \\ Mao Cai \\ Wenzhou Medical University Second Affiliated Hospital

\section{Chongjie Huang} \\ Wenzhou Medical University Second Affiliated Hospital \\ Dansi Qi \\ Wenzhou Medical University Second Affiliated Hospital \\ Changjia Li \\ Weihai Municipal Hospital \\ Long Li \\ Qingdao Municipal Hospital Group \\ Wanle Hu ( $\nabla$ wanlehu@126.com ) \\ Wenzhou Medical University Second Affiliated Hospital \\ Yaojun Yu \\ Wenzhou Medical University Second Affiliated Hospital
}

Research

Keywords: colorectal cancer, epigenetic, prognostic biomarker, prognosis

Posted Date: September 24th, 2020

DOl: https://doi.org/10.21203/rs.3.rs-76992/v1

License: (a) (i) This work is licensed under a Creative Commons Attribution 4.0 International License. Read Full License 


\section{Abstract}

Background

The $\mathrm{CpG}$ island methylator phenotype(CIMP)with extensive promoter methylation isa distinct epigenotype incolorectal cancer (CRC). Changes in microbiota and epigenetic dysregulation mightbe the key underlying mechanism.

Methods

Tissueswith stages I-III cancerwere collected afterproctocolectomy. The 16S rRNA gene sequencing was carried to determine the differences in microbiota.Subsequently,BRAF mutation,the statusof microsatellite instability (MSI, also known as mismatch repair deficiency) and CIMP were also tested. The Chi-square test was carriedoutto analyze the relationship between molecular changes (MSI and CIMP) and the development of CRC.

Results

Patients in the three groups differed in thetumorlocation $(P=0.034)$ and the carcinoembryonic antigen (CEA) level $(P=0.036)$. The positive CIMP and MSI-LOW/MSS were more common in the worse prognosis groups. The Kaplan-Meier and Cox proportional regression analyses indicated that CIMP and MSI were the independent indicators of poor survival since the positive rates of which were significantly higher in the non-survival group. Besides, there were differences in microbiota among tumor tissues of different prognoses, with the Fusobacterium nucleatum and Bacteroides fragilis more abundant in the worse prognosis groups.

Conclusion

The persistent epigenetic changes influence the prognosis of patients with CRC and the composition of the gut microbiota might be the cause.

\section{Background}

Colorectal cancer (CRC) is one of the most common malignancies worldwide, with over half a million deaths per year[1, 2].It represents a heterogeneous process with a differ ingset of somatic molecular alteration[3].It has been well demonstrated that the accumulation of both genetic and epigenetic alterations cancontribute to malignant transformation of normal colonic mucosa, leading to the development of CRC. Two molecular pathways have been reported: the microsatellite instability (MSI), and the $\mathrm{CpG}$ island methylator phenotype (CIMP) [4-7].MSI is caused by a defective mismatch repair system,resulting in an alteration in the number of repeated nucleotide(s), which causes frame-shift mutations of the MMR genes (MLH1, MSH2, MSH6, and PMS2) or the germ line deletion of the EPCAM gene[8, 9].

Genetic alterations and epigenetic changes, such as global and regional hypermethylation of CpG islands are the hallmarks of human cancer, which may result in the activation of oncogenes and the inactivation of tumor-suppressor genes[10-12].A subgroup of CRC with frequent aberrant DNA methylations of the CpG island has been reported, referred to as the CIMP. Currently, 2 panels of CIMP marker genes were used: the classic panel (MINT1, MINT2, MINT31, CDKN2A, and hMLH1) defined by Toyota et al.[13] and the new panel(CACNA1G, IGF2, NEUROG1, RUNX3, and SOCS1) defined by Weisenberger et al[14].According to the number of methylated promoters, tumors were classified as CIMP positive or CIMP negative. It has been well demonstrated that CIMP induced CRCs have distinct clinical and histological features, such as female predominance, proximal location, frequent BRAF mutations, and MSI[15-18].

In the past few years, with the prevailing of the16SrRNA gene sequencing approach, we could analyze the microbial community more effectively. So far, Microbiota has been a subject in intense scholarly debate. Accumulating evidence indicates that the Fusobacterium species (a group of nonspore-forming, anaerobic gram-negative bacteria) has a positive association with the status of CIMP and MSI[19]. 
Based on the above information, we aimto determine whether the CIMP status provides further insight into clinical behavior and survival in patients with CRC. Since the CIMP status associates with MSI and mutation of BRAF, we have quantified the expression levels in tumor tissues. Besides, we have also checked the structure of the microbiota community and the status of MSI and CIMP, and the mutation of the BRAF.It is expected that this study can reveal the pathological process of how theabove molecular phenotypes could affect the prognosis of CRC patients.

\section{Method}

\section{Study population and design}

A total of 180 individuals scheduled for colorectal resection at Wenzhou Medical University, Wenzhou, China, were recruited to the study. All patients with stages I-III cancer underwent a standard curative surgery, while stage IV CRC tissues were collected from patients who received palliative surgery to relieve serious cancer-related contradiction. The patients were not treated with antibiotics in the month before surgery but were administered antibiotics intravenously in a few hours after resection. Patients who had received or regularly used non-steroidal anti-inflammatory drugs, statins, or probiotics were excluded. Besides, those who had chronic bowel disease, infections, food allergies, and dietary restrictions were also excluded. Surgeries were performed at the Affiliated Hospital of Wenzhou Medical University between 2014 and 2017, and the treatments were given according to the National Comprehensive Cancer Network Guidelines.

\section{Sample preparation}

Tumor samples were obtained from the 180 patients who had undergone proctocolectomy. These CRC tissue samples and adjacent normal tissue samples (at least $5 \mathrm{~cm}$ from the tumor site) of these 180 patients were obtained from the gastrointestinal cancer specimen bank of the Affiliated Hospital of Wenzhou University. To be specific, surgically resected specimens were collected immediately after tumor removal and stored at $-80^{\circ} \mathrm{C}$. The TNM stages were determined according to the American Joint Committee on Cancer system and all specimens were graded histologically according to the World Health Organization classification criteria. Written informed consents of joining the specimen bank were obtained from all the patients before surgery, and the protocols used in the study were approved by the Ethics Committee of Affiliated Hospital of Wenzhou Medical University. Clinical and pathologic data were reviewed from the gastrointestinal cancer database of Affiliated Hospital of Wenzhou Medical University.

\section{DNA extraction}

CTAB method was used to extract DNA from all tumor samples with minimal modification. The concentration of DNA was measured by fluorometer or microplate reader and sample integrity was tested by agarose gel electrophoresis ( $1 \%$ concentration of agarose Gel; $150 \mathrm{~V} ; 40$ min electrophoresis time). All DNA samples were stored at $-20^{\circ} \mathrm{C}$.

\section{PCR and sequencing analysis}

The V4 region of the bacterial 16S rRNA gene was amplified by polymerase chain reaction (PCR) using universal primers 319F and 806R. The reaction mix consisted of Phusion High-Fidelity PCR Master Mix (NEB, Ipswich, MA, USA) and appropriate primer/probe pairs. The PCR program was as follows: Denaturation at $98^{\circ} \mathrm{C}$ followed by 30 cycles of $45 \mathrm{~s}$ at $95^{\circ} \mathrm{C}$ (denaturation) in $3 \mathrm{~min}$, thenannealing at $55^{\circ} \mathrm{C}$ and $45 \mathrm{~s}$ at $72^{\circ} \mathrm{C}$ (extension) in $45 \mathrm{~s}$, with a final extension at $72^{\circ} \mathrm{C}$ for 7 min. The PCR products were purified with AMPure XP beads (Agencourt Bioscience) to remove the unspecific products before library construction. The library was quantitated in two ways. The average molecule length was measured using the Agilent 2100 bioanalyzer instrument (Agilent DNA 1000 Reagents) and then quantified by real-time quantitative PCR (qPCR; EvaGreen TM). The sequencing of qualified libraries was performed at the BGI-Huada Genomics institute in Shenzhen using MiSeq System, with the sequencing strategy PE250 (PE251+8+8+251) or PE300 (PE301+8+8+301) (MiSeq Reagent Kit).

\section{Bioinformatics analysis}


The sequences were clustered into operational taxonomic units (OTUs) with a 97\% threshold using USEARCH (v7.0.1090) [20],thus the OTU unique representative sequences were obtained. Chimeras were filtered out using UCHIME (v4.2.40)[21]. Representative OTUs were aligned to the optimized sequences and the abundance of OTUs per sample was obtained for further analysis. Ribosomal Database Project (RDP) Classifier v.2.2 was used to taxonomically classify OTU representative sequences in the following databases: Greengene V201305[21] and RDP (Release9 201203)[22].

\section{Promoter Methylation Analysis}

The Definition of $\mathrm{CpG}$ is the land promoter hypermethylation of at least 2 out of 5 methylation markers (CACNA1G, CDKN2A, NEUROG1, CRABP1and MLH1), as proposed by Weisenberger et al[23]., to detect CIMP in tumor tissue of CRC. Methylation of these markers was determined by bisulfite modification of $500 \mathrm{ng}$ genomic DNA using a commercially available kit (Zymo Research), to create a methylation index with the CIMP markers and subsequent methylation-specific PCR (MSP) [24, 25]. Since MSP is effective, specific, and simple, it was used to detect CIMP. Also, the research shows that there are no differences between MSP and other technologies such as MethyLight[26].

\section{MSI Analysis}

As a second-generation genetic marker, MSI has been widely applied in tumor gene diagnosis and genetic analysis because of its high polymorphism, stability, and Mendelian co-dominant inheritance. It is determined through the multiplex fluorescent PCR

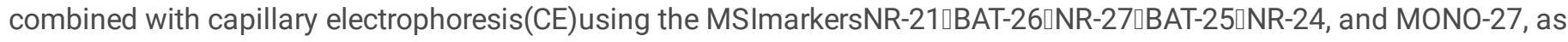
reported by Suraweera et al[27]. This method has high efficiency, stability, high sensitivity, thus it provides reliable analysis results. After amplified by Multiplex fluorescent PCR, the CE method using dual internal standards (molecular weight markers shorter and longer than the PCR fragment of interest) was implemented to measure microsatellite length. To validate the CE method in detecting MSI, a human tumor sample and a control DNA sample collected from the patients were also tested.

\section{Statistical analysis}

Statistical analyses were conducted using the SPSS 20 statistical software. The two-sided v2 test was used to determine the associations between CIMP status and different clinicopathological features. The significance was determined using the logrank test. Cox proportional hazard models were used to carry out multivariate survival analyses. Metastases (http://metastats.cbcb.umd.edu/) and R (v3.0.3) were used to determine which taxonomic groups were significantly different. The obtained $P$ values were adjusted by a Benjamini-Hochberg false discovery rate (FDR) correction (function 'P.adjust' in the stats package of $\mathrm{R}(\mathrm{v} 3.0 .3))$. $\mathrm{P} \leq 0.05$ was considered significant[28].

\section{Result}

\section{Associations between Molecular phenotype and clinicopathologic characteristics}

The clinical characteristics of the patients with different molecular phenotypes were compared, as shown in Table 1.Wecould see that the patients belonging to different groups might be correlated with the patients' outcomes in $\mathrm{CRC}$. Based on the stages of the tumor samples, we found that tumor location $(P=0.022)$, differentiation $(P=0.047)$, and tumor size $(P=0.031)$ were significantly associated with CIMP status, Besides, correlations between age $(P=0.026)$, gender $(P=0.045)$, remote Metastasis $(P$ $=0.005)$ and $\mathrm{MSI}$ status were also significant. In addition, gender $(P=0.046)$ and differentiation $(P=0.043)$ were found associated with BRAF mutation(Table 1). 
Table 1

Correlation between clinicopathological features and CIMP combined with MSI status

\begin{tabular}{|c|c|c|c|c|c|c|c|c|c|c|c|}
\hline \multicolumn{2}{|l|}{ Variable } & \multirow[t]{2}{*}{$\begin{array}{l}N \\
(180)\end{array}$} & \multicolumn{2}{|l|}{ CIMP } & \multirow[t]{2}{*}{$\begin{array}{l}P \\
\text { value }\end{array}$} & \multicolumn{2}{|l|}{ MSI } & \multirow{2}{*}{$\begin{array}{l}\mathrm{P} \\
\text { value } \\
-\mathrm{H}(42)\end{array}$} & \multicolumn{2}{|l|}{ BRAF } & \multirow[t]{2}{*}{$\begin{array}{l}P \\
\text { value }\end{array}$} \\
\hline & & & $\begin{array}{l}(-) \\
(145)\end{array}$ & $\begin{array}{l}(+) \\
(35)\end{array}$ & & $\begin{array}{l}\text { MSS/MSI- } \\
\text { L(138) }\end{array}$ & MS & & $\begin{array}{l}(-) \\
(129)\end{array}$ & $\begin{array}{l}(+) \\
(51)\end{array}$ & \\
\hline \multirow[t]{2}{*}{ Age } & $<60$ & 72 & 60 & 12 & 0.442 & 49 & 23 & $0.026^{*}$ & 55 & 17 & 0.251 \\
\hline & $>=60$ & 108 & 85 & 23 & & 89 & 19 & & 74 & 34 & \\
\hline \multirow[t]{2}{*}{ Gender } & Male & 109 & 87 & 22 & 0.756 & 78 & 31 & $0.045^{\star}$ & 84 & 25 & $0.046^{\star}$ \\
\hline & Famale & 71 & 58 & 13 & & 60 & 11 & & 45 & 26 & \\
\hline \multirow[t]{3}{*}{ Location } & L Colon & 37 & 24 & 13 & $0.022^{*}$ & 24 & 13 & 0.218 & 22 & 15 & 0.077 \\
\hline & R Colon & 32 & 26 & 6 & & 25 & 7 & & 21 & 11 & \\
\hline & Rectal & 111 & 95 & 16 & & 89 & 22 & & 86 & 25 & \\
\hline \multirow{2}{*}{$\begin{array}{l}\text { Lymphovascular } \\
\text { invasion }\end{array}$} & No & 122 & 101 & 21 & 0.148 & 98 & 24 & 0.092 & 92 & 30 & 0.106 \\
\hline & Yes & 58 & 44 & 14 & & 40 & 18 & & 37 & 21 & \\
\hline \multirow[t]{2}{*}{ Differentiation } & Poor & 48 & 34 & 14 & $0.047 *$ & 32 & 16 & 0.056 & 29 & 19 & $0.043^{*}$ \\
\hline & Well & 132 & 111 & 21 & & 106 & 26 & & 100 & 32 & \\
\hline \multirow[t]{2}{*}{ Tumor size } & $\geq 5 \mathrm{~cm}$ & 69 & 50 & 19 & $0.031 *$ & 52 & 17 & 0.744 & 55 & 14 & 0.059 \\
\hline & $\nabla 5 \mathrm{~cm}$ & 111 & 95 & 16 & & 86 & 25 & & 74 & 37 & \\
\hline \multirow[t]{2}{*}{ TNM stage } & I and II & 44 & 35 & 9 & 0.846 & 31 & 13 & 0.165 & 27 & 17 & 0.081 \\
\hline & III and IV & 136 & 110 & 26 & & 107 & 29 & & 102 & 34 & \\
\hline \multirow{2}{*}{$\begin{array}{l}\text { Remote } \\
\text { Metastasis }\end{array}$} & Nagetive & 171 & 140 & 31 & 0.073 & 135 & 34 & $0.005^{\star}$ & 121 & 50 & 0.239 \\
\hline & Positive & 9 & 5 & 4 & & 3 & 6 & & 8 & 1 & \\
\hline
\end{tabular}

\section{Cpg Island Methylator Phenotype (cimp) And Microsatellite Instability (msi}

After detecting the BRAF mutation and the status of MSI and CIMP, we compared these molecular features in the four groups. The results show that We could observe the relationship between different patients and CIMP status. Also, the difference could observe in MSI status. And those patients who have the statistically significant had the more positive rate in the non-survival group(Table 2) 
Table 2

\begin{tabular}{|c|c|c|c|c|c|c|c|}
\hline \multicolumn{2}{|l|}{ Variable } & \multirow[t]{2}{*}{$\begin{array}{l}N \\
(180)\end{array}$} & \multirow{2}{*}{$\begin{array}{l}\begin{array}{l}\text { survival } \\
\text { group }\end{array} \\
(\mathrm{N}=89)\end{array}$} & \multirow{2}{*}{$\begin{array}{l}\text { non-survival } \\
\text { group } \\
(\mathrm{N}=28)\end{array}$} & \multirow{2}{*}{$\begin{array}{l}\text { recurrence } \\
\text { group } \\
(\mathrm{N}=34)\end{array}$} & \multirow{2}{*}{$\begin{array}{l}\begin{array}{l}\text { unclear } \\
\text { group }\end{array} \\
(\mathrm{N}=29)\end{array}$} & \multirow[t]{2}{*}{$\begin{array}{l}\mathrm{P} \\
\text { value }\end{array}$} \\
\hline & & & & & & & \\
\hline \multirow[t]{2}{*}{ B-raf } & Negative & 128 & 66 & 20 & 24 & 18 & 0.668 \\
\hline & Positive & 52 & 23 & 8 & 10 & 11 & \\
\hline \multirow[t]{2}{*}{ CIMP } & Negative & 132 & 69 & 18 & 19 & 26 & $0.011^{*}$ \\
\hline & Positive & 48 & 20 & 10 & 15 & 3 & \\
\hline \multirow[t]{2}{*}{ MSI } & $\begin{array}{l}\text { MSS/MSI- } \\
\text { low }\end{array}$ & 139 & 70 & 17 & 25 & 27 & 0.031 \\
\hline & MSI-high & 41 & 19 & 11 & 9 & 2 & \\
\hline \multicolumn{8}{|c|}{ Bonferroni-correction: $a=0.017$} \\
\hline
\end{tabular}

\section{Prognostic value of CIMP,MSI, and BRAF}

The univariate Cox regression analyses revealed that positive CIMP(HR 1.914; $P=0.042), M S I(H R 2.549 ; P=0.002)$, TNM stage (HR 3.655; $P=0.006)$, and Lymph node metastasis (HR 1.940; $P=0.025)$ were associated with worse OS in CRC patients after radical surgery(FIGURE 1). However, only positive CIMP(HR 2.166; 95\% Cl 1.119,4.191; $\mathrm{P}=0.022)$ and TNM stage (HR 3.007; 95\% $\mathrm{Cl} 1.127,8.025 ; \mathrm{P}=0.028$ ) were independent predictors of the 3-year OS (Table 3). Furthermore, positive CIMP, MSI status,and differentiation were associated with poor 3-year DFS both in the univariate Cox regression analyses and the multivariate analysis (Table 4). 
Table 3

Univariate and multivariable Cox regression analyses for overall survival

\begin{tabular}{|lllllll|}
\hline \multicolumn{4}{|c}{ Univariate analysis } & \multicolumn{3}{c|}{ Multivariate analysis } \\
\hline Factor & HR & $95 \% \mathrm{Cl}$ & $\mathrm{p}$ & $\mathrm{HR}$ & $95 \% \mathrm{Cl}$ & $\mathrm{p}$ \\
\hline Age $(<60 />=60)$ & 0.554 & $(0.304,1.009)$ & 0.053 & & & \\
\hline Gender(male/female) & 1.073 & $(0.596,1.929)$ & 0.814 & & & \\
\hline Location(colon) & 0.818 & $(0.343,1.951)$ & 0.651 & & & \\
\hline Location(rectum) & 1.063 & $(0.524,2.158)$ & 0.865 & & & \\
\hline Tumor size & 0.713 & $(0.393,1.293)$ & 0.265 & & & \\
\hline (<5 cm/>=5 cm) & & & & & & \\
\hline lymph node metastasis (Yes/No) & 1.94 & $(1.086,3.467)$ & $0.025^{*}$ & 1.587 & $(0.868,2.900)$ & 0.134 \\
\hline Grade of differentiation & 0.749 & $(0.415,1.351)$ & 0.336 & & & \\
\hline (well/poor) & & & & & & \\
\hline TNM stage (I-II/III-IV) & 3.655 & $(1.441,9.273)$ & $0.006^{*}$ & 3.007 & $(1.127,8.025)$ & $0.028^{*}$ \\
\hline CIMP(+) & 1.914 & $(1.024,3.577)$ & $0.042^{*}$ & 2.166 & $(1.119,4.191)$ & $0.022^{*}$ \\
\hline BRAF(+) & 0.907 & $(0.471,1.745)$ & 0.77 & & & \\
\hline MSI(+) & 2.549 & $(1.425,4.559)$ & $0.002^{*}$ & 1.845 & $(0.996,3.420)$ & 0.052 \\
\hline HR relative risk, 95\% Cl 95\% confidence interval. & & & & \\
\hline
\end{tabular}

Table 4

Univariate and multivariable Cox regression analyses for disease free survival

\begin{tabular}{|c|c|c|c|c|c|c|}
\hline \multirow[b]{2}{*}{ Factor } & \multicolumn{3}{|c|}{ Univariate analysis } & \multicolumn{3}{|c|}{ Multivariate analysis } \\
\hline & $\mathrm{HR}$ & $95 \% \mathrm{Cl}$ & $\mathrm{p}$ & $\mathrm{HR}$ & $95 \% \mathrm{Cl}$ & $\mathrm{p}$ \\
\hline $\operatorname{Age}(<60 />=60)$ & 0.527 & $(0.232,1.199)$ & 0.127 & & & \\
\hline Gender(male/female) & 0.87 & $(0.374,2.021)$ & 0.746 & & & \\
\hline Location(colon) & 1.316 & $(0.264,6.552)$ & 0.737 & & & \\
\hline Location(rectum) & 2.314 & $(0.676,7.917)$ & 0.181 & & & \\
\hline $\begin{array}{l}\text { Tumor size } \\
(<5 \mathrm{~cm} />=5 \mathrm{~cm})\end{array}$ & 0.637 & $(0.262,1.553)$ & 0.322 & & & \\
\hline lymph node metastasis (Yes/No) & 0.242 & $(0.057,1.035)$ & 0.056 & & & \\
\hline $\begin{array}{l}\text { Grade of differentiation } \\
\text { (well/poor) }\end{array}$ & 4.596 & $(1.073,19.688)$ & $0.040 *$ & 4.403 & $(1.021,18.994)$ & $0.047^{*}$ \\
\hline TNM stage (I-II/III-IV) & 0.452 & $(0.198,1.031)$ & 0.059 & & & \\
\hline $\operatorname{CIMP}(+)$ & 3.263 & $(1.373,7.752)$ & $0.007^{\star}$ & 3.130 & $(1.305,7.503)$ & $0.011 *$ \\
\hline $\operatorname{BRAF}(+)$ & 0.843 & $(0.331,2.144)$ & 0.719 & & & \\
\hline $\mathrm{MSI}(+)$ & 3.637 & $(1.495,8.846)$ & $0.004^{\star}$ & 2.985 & $(1.198,7.434)$ & $0.019 *$ \\
\hline
\end{tabular}




\section{Diversity and structural changes of the tumor microbiota in CRC patients with different prognosis outcomes}

The overall microbiota at the phylum level is shown in Fig. 2A. The dominant phyla in all groups were Proteobacteria (33.8\%-49.4\%), Firmicutes (16.9\%-22.7\%), Bacteroidetes (21.1\%-27.9\%), and Fusobacterium (3.38\%-10.8\%). When comparing the relative abundance, we found that the abundance of Proteobacteria was higher in the survival group than in non-survival group $(48.2 \%$ vs. $33.8 \%$, FDR $=0.063)$, while Fusobacterium was lower in survival group $(3.38 \%$ vs. $9.71 \%, F D R=0.089)$, although the differences were not statistically significant.

The microbial composition was different at the genus level among groups. Shewanella (9.05\% vs. 5.76\%, FDR = 0.091), Methylobacterium $(2.54 \%$ vs. $1.56 \%$, FDR $=0.039)$, Faecalibacterium $(2.99 \%$ vs. $0.93 \%$, FDR $=0.016)$, and Sphingomonas $(1.38 \%$ vs. $0.79 \%, F D R=0.031$ ) together account over $1 \%$ of the total bacteria in the survival group, exhibiting a relatively higher abundance than that in non-survival group. While Fusobacterium ( $9.23 \%$ vs. $2.70 \%$, FDR $=0.079$ ) was relatively more abundant in the non-survival group and the difference was borderline significant. Methylobacterium $(2.54 \%$ vs. $1.51 \%, F D R=0.09)$ and Mycoplasma $(0.64 \%$ vs. $0 \%$, FDR $=0.01)$ showed higher abundance in survival group(Fig. $2 \mathrm{~B})$.

In specie level, we found a higher level of $B$. fragilis ( $9.75 \%$ vs. $2.62 \%, F D R=0.017)$ in non-survival group than in survival group, while F. prausnitzii $(2.96 \%$ vs. $0.92 \%, \mathrm{FDR}=0.028)$ and Methylobacteriumsuomiense $(1.91 \%$ vs. $0.78 \%, \mathrm{FDR}=0.098)$ were more abundant in the survival group. Moreover, borderline statistic difference was found in F. nucleatum between non-survival group and survival group $(5.66 \%$ vs. $1.08 \%, \mathrm{FDR}=0.076)$ and $F$. nucleatum $(5.10 \%$ vs. $1.08 \%$, FDR $=0.08)$ exhibited a greater abundance in the recurrence group than in the survival group (Fig. $2 \mathrm{C}$ ).

\section{Discussion}

CIMP status is an important indicator to show the development of CRC. Recent studies have shown that through changing epigenetic, namely changes in gene expression patterns, CIMP can cause the occurrence of CRC mediated by DNA methylation transferase and S-adenosine methionine[29-31]. Acid provides a methyl group, and a methyl group is added to the fifth carbon atom of cytosine, making a chemical modification reaction of 5-methylcytosine. This process mainly occurs on CpG dinucleotides,thus epigene silencing is caused by DNA methylation. CIMP, as an epigenetic phenotype of CRC, has different clinical, pathological, and biological characteristics, such as being related to proximal colon, female, poor differentiation, MSI, BRAF high-frequency mutations, tp53 low-frequency mutations[32-34], and CIMP-H, MSI-H and TGFBR2 single nucleotide mutation. During the development of CRC, CIMP that progresses to dentate adenomas shows frequent promoter methylation, while flat adenomas not[35].

To our best knowledge, this is the first study to compare the CIMP status, MSI,and BRAF among groups of cancer tissues divided by different post-operation prognoses. CIMP has been extensively studied in CRC because it reflects epigenetic aberration in tumor cells[36, 37]. CIMP, as an epigenetic phenotype of CRC, has different clinical, pathological, and biological characteristics, such as related to proximal colon, female, poor differentiation, this was also demonstrated in our study. Divergent findings ofthe prognostic values of CIMP have been reported. Some studies suggest an adverse effect of CIMP on the survival of CRC patients[38-42], whereas other studies reported no relationship between CIMP status and prognosis in CRC[4346]. These contradictory results can be explained at least in part by the absence of a consensus CIMP panel since some studies used the classical panel defined by Toyota et al[37]., whereas others used the new CIMP panel[13]. Overall, MSI often manifests as a Pathological type with poor prognoses such as poor differentiation or mucinous adenocarcinoma[47], and remote metastasis was also found in our study. Besides, we also examined the BRAF mutation on patients, CIMP-high status was independently associated with a bad differential, whereas BRAF mutation was associated with a significantly increasing numbers. It might explain why these two molecular phenotypes were associated with bad outcomes in many studies[48, 49]. 
The specific mechanisms by which gut microbiota affects the development of CRC are still not well understood. One of the most promising theories is the microbe-driven intestinal mechanism. Interestingly, F. nucleatum, B. fragilis, and F. prausnitzii are all key players in modifying intestinal inflammation levels. To our knowledge, CIMP is believed to promote carcinogenesis through methylation mediated transcriptional silencing in tumor suppressor genes[32]. However, the role of microbiota in the progress of CIMP and the mechanism underlying its sustained influence remains unknown. In fact, the relationships between CIMP, MSI, BRAF mutations, and the gut microbiota in colorectal cancer arecomplex. To decipher the complex association of CIMP, MSI, and BRAF mutation on patients' microbiota, we collect the patients' CRC tissues in four groups. The results showed the relationship in CIMP status between different groups, which was consistent with previous studies. Besides, the higher incidence was observed in there currence group, with a greater abundance of $F$. nucleatum. Some studies have shown that $F$. nucleatum is associated with epigenetic changes such as the status of MSI and CIMP[50], which also corresponds with the results in this study. In addition, another study has also shown that patients with a high level of $F$. nucleatum have a significantly shorter survival time, which was similarly obtained in this study[51]. But their sample size was relatively small. A more recent study has reported a similar result using a larger database of CRC cases in the USA, revealing a correlation between a high amount of tissue $F$. nucleatum DNA and higher CRC-specific mortality. Evidence has also shown that overexpression of $B R A F$ were markers of the poor prognosis[52,53], Moreover, MSI, the primary causes of which is hypermethylation of the MLH1 promoter, was also associated with the clinical outcomes of $\mathrm{CRC}[4,6]$.A relevant study hasshown that microbiota has a relation with MMR, which is the most important mechanism for the appearance of MSI[54]. Furthermore, overexpression of BRAF was also considered the marker of poor prognosis which was consistent with our findings of prognostic values of $F$. nucleatum, $B$. fragilis,and F.prausnitzi ${ }^{[55]}$. This might explain why BRAF mutation could accompany MSI as reported in the previous studies.

Herein, we classified tumors according to the CIMP status combined with BRAF methylation or MSI status to investigate whether these molecular characteristics affect clinical behavior and prognosis. Our data showed that clinicopathologic features and OS differed among the 4 groups of patients according to their CIMP status combined with BRAF methylation or MSI status. In fact, CIMP and BRAF tests are used to exclude HNPCC among patients who exhibit MSI-high, since HNPCC seldom exhibits CIMP or BRAF mutation. Therefore, studies on epigenetic and/or genetic alterations areincreasingly important in cancer research. As we all know, CIMP-H is associated with poor OS, but some reports suggested that MSI-H coulddecrease this phenomenon, which explains why we could see better outcomes when the CIMP-H was companiedwith the MSI-H. The limited sample sizemightalso be an important reason why we couldn't see the statisticaldifferencewhen we were analyzingthe data.Studies have shown that $70-80 \%$ of MSI induced CRC can be attributed to CIMP related MLH1 methylation. The mechanism is that CIMP hypermethylation leads to methylation of the MLH1 promoter, which results in the silencing of the MLH1 mismatch repair gene and the final performance MSI status.Some previous studies on the correlation between microsatellite status, CIMP status, and OS have shown that CIMP positive is negatively correlated with OS.In MSI patients, CIMP and OS also show a certain correlation. Ward et al. found that CIMP positive was associated with the total OSbut not survival time, and Rhee et al. also proved this. In this study, a similar conclusion wasconfirmed.

Finally, it is important to mention that our study had some limitations. The major one is the small size after dividingthe cohort into 4 subgroups. Although we have studied the related risk factors and survival rate, we have not explored the correlation between these phenomena and cancer medication.Besides, some mechanisms between them and the intestinal micro-ecology are just a taste.

\section{Conclusions}

Our results underline the interest that CIMP can be useful to predict prognosis in CRC patients, and at present, it is believed that $\mathrm{MSI}$ is closely related to the occurrence and development of CRC. So far, MSI has beena tumor-related factor recognized worldwide. Microbiota, such as B. fragilis and F. prausnitzii, participate in influencing the course/progression of CRC in patients subjected to energy restriction in their early childhood, which further validates the association of $F$. nucleatum with epigenetic changes and gene mutations. The future goal is to use precision medicine to analyze the patient's genes, select the most suitable individualized treatment plan for the patient, and make the most accurate judgment on the patient's prognosis. 


\section{Abbreviations}

1. CIMP: CpG Island Methylator Phenotype

2. CRC: colorectal cancer

3. CEA:carcinoembryonic antigen

4. PCR:polymerase chain reaction

5. FDR:false discovery rate

\section{Declarations}

The authors do not have any conflicts of interest with the content of the manuscript.

\section{ETHICS APPROVAL AND CONSENT TO PARTICIPATE}

The ethical committee approved the procedure.

\section{CONSENT FOR PUBLICATION}

Written informed consent for publication was obtained from all participants.

\section{AVAILABILITY OF DATE AND MATERIALS}

The raw/processed data required to reproduce these findings cannot be shared at this time as the data also forms part of an ongoing study.

\section{COMPETING INTERESTS}

None.

\section{FUNDING}

This work was supported by grants from Zhejiang Provincial Department of Health Project (grant Y20170174), Natural Science Foundation of Zhejiang Provincial, China (grant 2017KY476).

\section{AUTHORS' CONTRIBUTIONS}

JTL YJY and WLH plan the study made all coordination and was involved in the laboratory processing. $\mathrm{KPH}, \mathrm{CCW}, \mathrm{MC}$ and CJH participated in the study and performed the statistical analysis. DSQ, CJL and LL carried out handling the samples. All authors read and approved the final version of manuscript.

\section{ACKNOWLEDGEMENTS}

This thesis would not have been possible without the consistent and valuable reference materials that I received from my Superior doctor $\mathrm{Hu}$, whose insightful guidance and enthusiastic encouragement in the course of my shaping this thesis definitely gain my deepest gratitude.

\section{References}

[1] Jemal A. Global Cancer Statistics. Cancer. 2011. 6(2): 69-90.

[2] Bray F, Ren JS, Masuyer E, Ferlay J. Global estimates of cancer prevalence for 27 sites in the adult population in 2008 . Int J Cancer. 2013. 132(5): 1133-45. 
[3] Ohm SBBaJE. Epigenetic gene silencing in cancer - A mechanism for early oncogenic pathway addiction. Cancer. 2006. 6: 107-116.

[4] Saadallah-Kallel A, Abdelmaksoud-Dammak R, Triki M, et al. Clinical and prognosis value of the CIMP status combined with MLH1 or p16 INK4a methylation in colorectal cancer. Med Oncol. 2017. 34(8): 147.

[5] Chung DC. The genetic basis of colorectal cancer: insights into critical pathways of tumorigenesis. Gastroenterology. 2000. 119(3): 854-65.

[6] Ogino S, Kawasaki T, Kirkner GJ, Suemoto Y, Meyerhardt JA, Fuchs CS. Molecular correlates with MGMT promoter methylation and silencing support CpG island methylator phenotype-low (CIMP-low) in colorectal cancer. Gut. 2007. 56(11): 1564-71.

[7] Thibodeau SN. Microsatellite instability in colorectal cancer: Different mutator phenotypes and the principal involvement of hMLH1. Cancer Res. 1998. 58: 1713-1718.

[8] Kim JH, Shin SH, Kwon HJ, Cho NY, Kang GH. Prognostic implications of CpG island hypermethylator phenotype in colorectal cancers. Virchows Arch. 2009. 455(6): 485-94.

[9] Verrico FZ\&LR\&M. Focus on genetic and epigenetic events of colorectal cancer pathogenesis: Implications for molecular diagnosis. Tumor Biol. 2014. 10.

[10] Li J WZ, Cao S. A pilot study on clinicopathological features and intestinal microflora changes in colorectal cancer patients born over a nine-year period encompassing three years before and after the Great Chinese famine. Cancer Epidemiol. Cancer Epidemiol. 2019. 59: 166-172.

[11] Issa J. CpG island methylator phenotype in cancer. NATURE REVIEWS | CANCER. 2004. 4(12): 988-93.

[12] Berg M, Søreide K. Genetic and epigenetic traits as biomarkers in colorectal cancer. Int J Mol Sci. 2011. 12(12): $9426-39$.

[13] Toyota M. Distinct genetic profiles in colorectal tumors with or without the CpG island methylator phenotype. Proc Natl Acad Sci U S A. 2002. 97(2): 710-715.

[14] Weisenberger DJ. CpG island methylator phenotype underlies sporadic microsatellite instability and is tightly associated with BRAF mutation in colorectal cancer. Nat Genet. 2006. 38(7): 787-793.

[15] Haixing JV. CpG island methylator phenotype in sporadic colorectal cancers and its relationship with microsatellite instability. CHINA MODERN DOCTOR. 2013. 51(26): 51-53.

[16] van Rijnsoever M, Grieu F, Elsaleh H, Joseph D, lacopetta B. Characterisation of colorectal cancers showing hypermethylation at multiple CpG islands. Gut. 2002. 51(6): 797-802.

[17] Kambara T, Simms LA, Whitehall VL, et al. BRAF mutation is associated with DNA methylation in serrated polyps and cancers of the colorectum. Gut. 2004. 53(8): 1137-44.

[18] Wei Z, Cao S, Liu S, et al. Could gut microbiota serve as prognostic biomarker associated with colorectal cancer patients' survival? A pilot study on relevant mechanism. Oncotarget. 2016. 7(29): 46158-46172.

[19] DeSantis TZ, Hugenholtz P, Larsen N, et al. Greengenes, a chimera-checked 16S rRNA gene database and workbench compatible with ARB. Appl Environ Microbiol. 2006. 72(7): 5069-72.

[20] Cole JR, Wang Q, Cardenas E, et al. The Ribosomal Database Project: improved alignments and new tools for rRNA analysis. Nucleic Acids Res. 2009. 37(Database issue): D141-5. 
[21] Zucker S, Vacirca J. Role of matrix metalloproteinases (MMPs) in colorectal cancer. Cancer Metastasis Rev. 2004. 23(1-2): 101-17.

[22] Derks S, Lentjes MH, Hellebrekers DM, de Bruine AP, Herman JG, van Engeland M. Methylation-specific PCR unraveled. Cell Oncol. 2004. 26(5-6): 291-9.

[23] JAMES G. H. Methylation-specific PCR: A novel PCR ssay for methylation status of CpG islands. Medical Sciences: Herman. 1996. 93: 9821-9826.

[24] Barault L, Charon-Barra C, Jooste V, et al. Hypermethylator phenotype in sporadic colon cancer: study on a population-based series of 582 cases. Cancer Res. 2008. 68(20): 8541-6.

[25] Suraweera N, Duval A, Reperant M, et al. Evaluation of tumor microsatellite instability using five quasimonomorphic mononucleotide repeats and pentaplex PCR. Gastroenterology. 2002. 123(6): 1804-11.

[26] White JR, Nagarajan N, Pop M. Statistical methods for detecting differentially abundant features in clinical metagenomic samples. PLoS Comput Biol. 2009. 5(4): e1000352.

[27] Waterland RA, Michels KB. Epigenetic epidemiology of the developmental origins hypothesis. Annu Rev Nutr. 2007. 27: 36388.

[28] Fraga MF, Ballestar E, Paz MF, et al. Epigenetic differences arise during the lifetime of monozygotic twins. Proc Natl Acad Sci U S A. 2005. 102(30): 10604-9.

[29] Feinberg AP. The epigenetic progenitor origin of human cancer. NATURE REVIEWS. 2006. 7: 21-33.

[30] Grady WM. Epigenetic events in the colorectum and in colon cancer. Biochem Soc Trans. 2005. 33(Pt 4): 684-8.

[31] Clarke MF. Epigenetic regulation of normal and cancer stem cells. Ann N Y Acad Sci. 2005. 1044: 90-3.

[32] Samowitz WS, Albertsen H, Herrick J, et al. Evaluation of a large, population-based sample supports a CpG island methylator phenotype in colon cancer. Gastroenterology. 2005. 129(3): 837-45.

[33] S Ogino MC, Kawasaki T. CpG island methylator phenotype (CIMP) of colorectal cancer is best characterised by quantitative DNA methylation analysis and prospective cohort studies. Gut. 2006. 55: 1000-1006.

[34] Ogino S, Kawasaki T, Kirkner GJ, et al. Down-regulation of p21 (CDKN1A/CIP1) is inversely associated with microsatellite instability and CpG island methylator phenotype (CIMP) in colorectal cancer. J Pathol. 2006. 210(2): 147-54.

[35] Ogino S, Kawasaki T, Ogawa A, Kirkner GJ, Loda M, Fuchs CS. TGFBR2 mutation is correlated with CpG island methylator phenotype in microsatellite instability-high colorectal cancer. Hum Pathol. 2007. 38(4): 614-20.

[36] Baylin SB, Ohm JE. Epigenetic gene silencing in cancer - a mechanism for early oncogenic pathway addiction. Nat Rev Cancer. 2006. 6(2): 107-16.

[37] Nagasaka T. Colorectal Cancer With Mutation in BRAF, KRAS, and Wild-Type With Respect to Both Oncogenes Showing Different Patterns of DNA Methylation. J Clin Oncol. 2004. 22(22): 4584-4594.

[38] 2005. Colorectal Cancers with the Uncommon Findings of KRAS Mutation and Microsatellite Instability. Cytogenet Genome Res. .

[39] Van Rijnsoever M, Elsaleh H, Joseph D, McCaul K, lacopetta B. CpG island methylator phenotype is an independent predictor of survival benefit from 5-fluorouracil in stage III colorectal cancer. Clin Cancer Res. 2003. 9(8): 2898-903.

[40] Phipps Al. Association Between Molecular Subtypes of Colorectal Cancer and Patient Survival. Gastroenterology. 2014.14. 
[41] Cha Y, Kim KJ, Han SW, et al. Adverse prognostic impact of the CpG island methylator phenotype in metastatic colorectal cancer. Br J Cancer. 2016. 115(2): 164-71.

[42] Barault L. Hypermethylator Phenotype in Sporadic Colon Cancer: Study on a Population-Based Series of 582 Cases. Cancer Res. 2008. 68(20): 8541-8546.

[43] Jia M, Jansen L, Walter V, et al. No association of $\mathrm{CpG}$ island methylator phenotype and colorectal cancer survival: population-based study. Br J Cancer. 2016. 115(11): 1359-1366.

[44] Zlobec I, Bihl MP, Foerster A, Rufle A, Lugli A. The impact of CpG island methylator phenotype and microsatellite instability on tumour budding in colorectal cancer. Histopathology. 2012. 61(5): 777-87.

[45] Li X, Hu F, Wang Y, et al. CpG island methylator phenotype and prognosis of colorectal cancer in Northeast China. Biomed Res Int. 2014. 2014: 236361.

[46] Jia M, Gao X, Zhang Y, Hoffmeister M, Brenner H. Different definitions of CpG island methylator phenotype and outcomes of colorectal cancer: a systematic review. Clin Epigenetics. 2016. 8: 25.

[47] Akiyama S, Kikuchi D, Mitani T, et al. A case of mucinous adenocarcinoma in the setting of chronic colitis associated with intestinal spirochetosis and intestinal stricture. Medicine (Baltimore). 2015. 94(4): e493.

[48] Berg M. Inffluence of Microsatellite Instability and KRAS and BRAF Mutations on Lymph Node Harvest in Stage I-III Colon Cancers. Mol Med. 2013. 19: 286-293.

[49] Ogino S, Kawasaki T, Kirkner GJ, Loda M, Fuchs CS. CpG island methylator phenotype-low (CIMP-low) in colorectal cancer: possible associations with male sex and KRAS mutations. J Mol Diagn. 2006. 8(5): 582-8.

[50] Mima K, Nishihara R, Qian ZR, et al. Fusobacterium nucleatum in colorectal carcinoma tissue and patient prognosis. Gut. 2016. 65(12): 1973-1980.

[51] Soucek LF\&JS\&ME\&P. Fusobacterium nucleatum associates with stages of colorectal neoplasia development, colorectal cancer and disease outcome. Eur J Clin Microbiol Infect Dis. 2014. 2018(3).

[52] Kumar R, Angelini S, Czene K, et al. BRAF mutations in metastatic melanoma: a possible association with clinical outcome. Clin Cancer Res. 2003. 9(9): 3362-8.

[53] Lucci-Cordisco E. The use of microsatellite instability, mmunohistochemistry and other variables in determining the clinical signifificance of MLH1 and MSH2 unclassifified variants in Lynch syndrome. Cancer Biomark. 2006. 26(201).

[54] Vlaykova T. Microsatellite instability and promoter hypermethylation of MLH1 and MSH2 in patients with sporadic colorectal cancer. Journal of B.u.on. Official Journal of the Balkan Union of Oncology. 2011. 16(2).

[55] Maija R.J. Kohonen-Corish JJD. Low Microsatellite Instability Is Associated With Poor rognosis in Stage C Colon Cancer. J Clin Oncol. 2006. 23(10): 2318-2324.

\section{Figures}



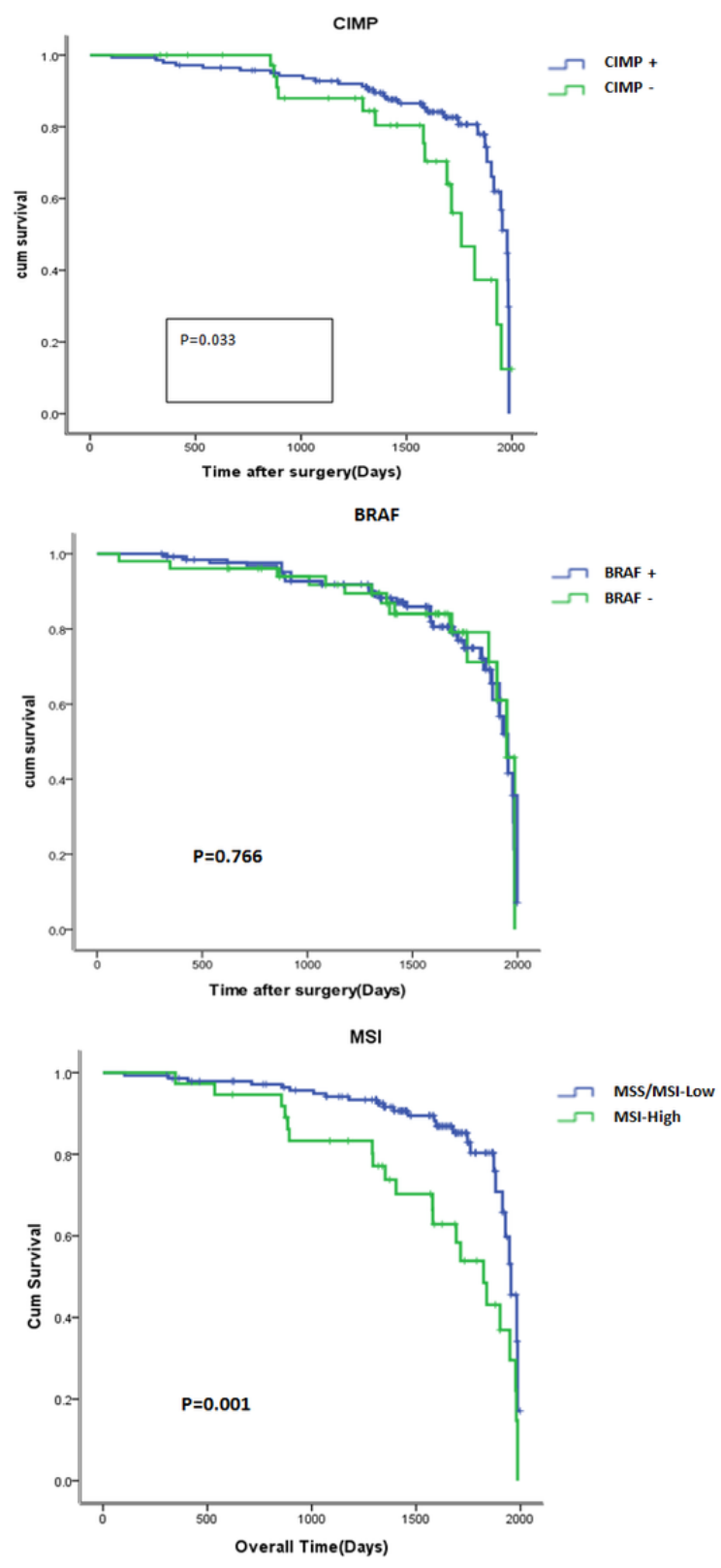

\section{Figure 1}

Kaplan-Meier survival curves for overall survival (OS) and disease free survival (DFS) in $180 \mathrm{CRC}$ patients in relation to CIMP, BRAF and MSI. P values were obtained by log-rank test. 

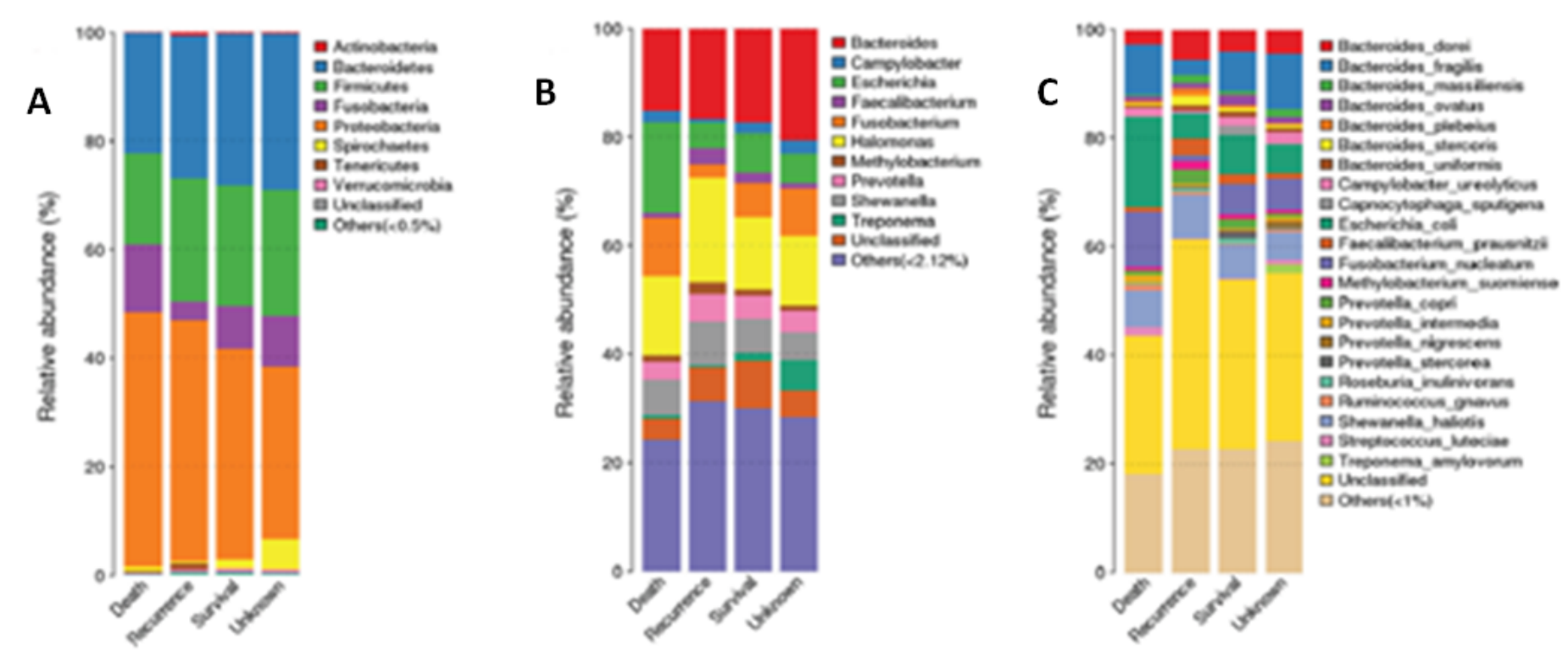

Figure 2

Diversity and structural changes of the tumor microbiota among the Non-survival group $(n=28)$, Recurrent group $(n=34)$, Survival group $(n=89)$ and Unclear group $(n=29)$. A. The dominant phyla of different groups. $B$. The dominant genera of different groups. C. The dominant species of different groups. 